\title{
CD45 ligation expands Tregs by promoting interactions with DCs
}

\author{
Geoffrey Camirand, ${ }^{1}$ Ying Wang,,${ }^{1}$ Yuning Lu, ${ }^{2}$ Yisong Y. Wan, ${ }^{3}$ Yan Lin, ${ }^{1}$ Songyan Deng, ${ }^{4}$ Galip Guz, ${ }^{4}$ David L. Perkins, ${ }^{5}$ \\ Patricia W. Finn, ${ }^{5}$ Donna L. Farber, ${ }^{6}$ Richard A. Flavell, ${ }^{7}$ Warren D. Shlomchik, ${ }^{4}$ Fadi C. Lakkis, ${ }^{1}$ \\ Christopher E. Rudd, ${ }^{2}$ and David M. Rothstein ${ }^{1}$
}

'Thomas E. Starzl Transplantation Institute, University of Pittsburgh School of Medicine, Pittsburgh, Pennsylvania, USA. Division of Immunology, Department of Pathology, University of Cambridge, Cambridge, United Kingdom. 'Lineberger Comprehensive Cancer Center, Department of Microbiology and Immunology, University of North Carolina at Chapel Hill, Chapel Hill, North Carolina, USA.

${ }^{4}$ Department of Medicine, Yale School of Medicine, New Haven, Connecticut, USA. ${ }^{5}$ Department of Medicine, University of Illinois College of Medicine, Chicago, Illinois, USA. ${ }^{6}$ Columbia Center for Translational Immunology, Columbia University Medical Center, New York, New York, USA. 'Department of Immunobiology and Howard Hughes Medical Institute, Yale School of Medicine, New Haven, Connecticut, USA.

\begin{abstract}
Regulatory T cells (Tregs), which express CD4 and FOXP3, are critical for modulating the immune response and promoting immune tolerance. Consequently, methods to expand Tregs for therapeutic use are of great interest. While transfer of Tregs after massive ex vivo expansion can be achieved, in vivo expansion of Tregs would be more practical. Here, we demonstrate that targeting the CD45 tyrosine phosphatase with a tolerogenic anti-CD45RB mAb acutely increases Treg numbers in WT mice, even in absence of exogenous antigen. Treg expansion occurred through substantial augmentation of homeostatic proliferation in the preexisting Treg population. Moreover, anti-CD45RB specifically increased Treg proliferation in response to cognate antigen. Compared with conventional T cells, Tregs differentially regulate their conjugation with DCs. Therefore, we determined whether CD45 ligation could alter interactions between Tregs and DCs. Live imaging showed that CD45 ligation specifically reduced Treg motility in an integrin-dependent manner, resulting in enhanced interactions between Tregs and DCs in vivo. Increased conjugate formation, in turn, augmented nuclear translocation of nuclear factor of activated T cells (NFAT) and Treg proliferation. Together, these results demonstrate that Treg peripheral homeostasis can be specifically modulated in vivo to promote Treg expansion and tolerance by increasing conjugation between Tregs and DCs.
\end{abstract}

\section{Introduction}

Regulatory $\mathrm{CD}^{+} \mathrm{T}$ cells expressing FOXP3 (Tregs) play a critical role in tolerance by modulating immune responses to both endogenous and exogenous antigens $(1,2)$. FOXP3 is required for Treg development and function, and FOXP3 deficiency leads to systemic autoimmunity (reviewed in refs. 1, 2). Tregs comprise approximately $10 \%$ of peripheral $\mathrm{CD} 4^{+}$cells and primarily arise as a distinct lineage in the thymus (natural Treg [nTreg]). However, peripheral FOXP3- conventional $\mathrm{CD}^{-}{ }^{+} \mathrm{T}$ cells (Tconvs) can convert into "induced" FOXP3 ${ }^{+}$Tregs (iTregs) in certain microenvironments, such as the gut, where they help maintain mucosal tolerance (3-5).

Given their dominant role in induction and maintenance of tolerance, there has been great interest in therapeutic manipulation of Tregs. The adoptive transfer of exogenous Tregs requires massive ex vivo expansion, and, despite recent progress, concerns over Treg purity and stability remain (6). Alternatively, tolerance may be enhanced by expanding Tregs in vivo. iTregs can be induced under specialized circumstances $(2,5,7-11)$. While this

Note regarding evaluation of this manuscript: Manuscripts authored by scientists associated with Duke University, The University of North Carolina at Chapel Hill, Duke-NUS, and the Sanford-Burnham Medical Research Institute are handled not by members of the editorial board but rather by the science editors, who consult with selected external editors and reviewers.

Conflict of interest: The authors have declared that no conflict of interest exists. Submitted: November 6, 2013; Accepted: August 6, 2014.

Reference information: J Clin Invest. 2014;124(10):4603-4613. doi:10.1172/JCI74087. can contribute to systemic tolerance, induction of iTregs during an active immune response has been observed primarily in TCR transgenic models. The extent to which this occurs in a polyclonal setting with typical antigen affinities remains unclear (12).

An approach that has received less attention is the specific expansion of preexisting Tregs. Endogenous or transferred Tregs in WT mice undergo significantly greater homeostatic proliferation (HP) than Tconvs, and Treg numbers are very sensitive to changes in proliferation or survival (13-16). Peripheral homeostasis is dependent on antigen presentation by DCs, since Treg number and HP are reduced when DCs lack MHC class II (17). Moreover, CD28 is required to maintain HP and peripheral Treg numbers $(16,18-20)$. This is only partially attributable to decreased IL-2 production by Tconvs in the absence of CD28 (18). In this regard, IL-2 neutralization decreases Treg proliferation and cell number and can precipitate autoimmunity $(14,21)$, whereas exogenous IL-2 can expand Tregs (as well as NK cells and cytotoxic T lymphocytes (22-24). How peripheral homeostasis of Tregs can best be targeted to promote tolerance remains to be determined.

We showed previously that anti-CD45RB treatment induces donor-specific tolerance in stringent murine allograft models, and this is dependent on Tregs and induction of CTLA- 4 on CD $4^{+}$cells (25-29). This raised the possibility that anti-CD45RB acts through generation of Tregs. We now show that anti-CD45RB nearly doubled both the frequency and number of endogenous Tregs in WT mice, due to specific enhancement of nTreg proliferation in response to antigen. Surprisingly, CD45 ligation preferentially 
A

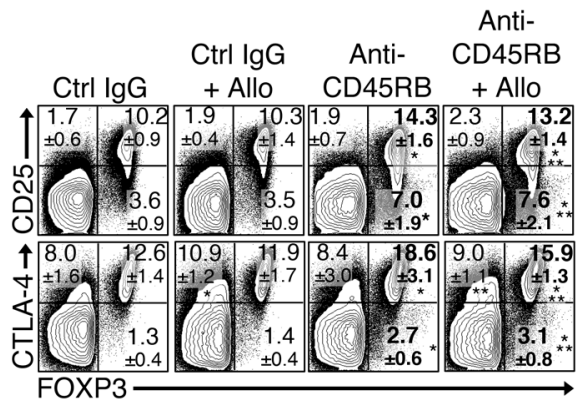

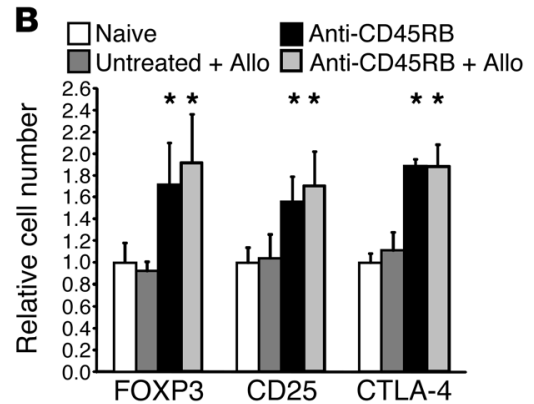
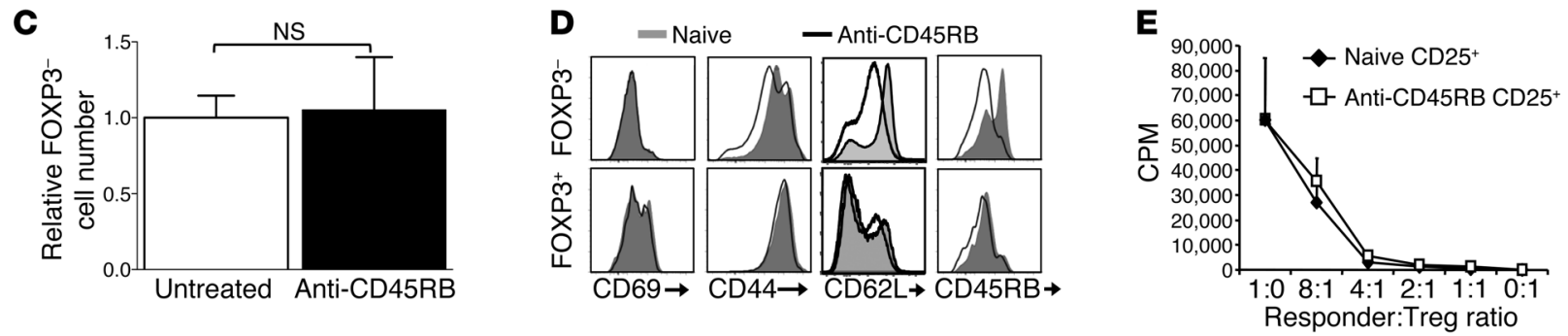

Figure 1. Anti-CD45RB treatment acutely induces FOXP3+ Tregs in the periphery. (A) Representative FOXP3, CTLA-4, and CD25 expression on day 10 by flow cytometry on splenic CD4+ $T$ cells from mice that received control IgG, alloantigen (Allo) and control IgG, anti-CD45RB (day -1, 0, and 5), or alloantigen and anti-CD45RB. Numbers represent mean percentage \pm SD expression in live CD4+ $T$ cells. ${ }^{*} P<0.05$ vs. control lgG, ${ }^{*} P<0.05$ vs. alloantigen and control lgG ( $n=8-19$ mice per group from 4 independent experiments). (B) Number of splenic FOXP3 ${ }^{+}$, CD25 , and CTLA-4+CD4+ cells in each group of mice on day 10 relative to that in naive untreated control mice ( $n=3-14$ mice per group). ${ }^{*} P<0.05$ vs. naive or untreated and alloantigen. (C) Number of FOXP3 ${ }^{-C D 4}{ }^{+}$cells from spleens of mice treated with anti-CD45RB (day 10) relative to that in untreated mice ( $n=8-10$ mice per group, from 3 independent experiments; NS, not significant). (D) Representative flow cytometry histograms of CD69, CD44, CD62L, and CD45RB expression on F0XP3 ${ }^{+}$and FOXP3-CD4 ${ }^{+}$cells from naive and anti-CD45RB-treated mice (day 10). (E) Representative in vitro suppression by Tregs from anti-CD45RB-treated and untreated mice. Sorted CD4 ${ }^{+} \mathrm{CD} 25^{+}$cells from naive (untreated) or anti-CD45RB-treated mice (day 10) were cultured in varying numbers with irradiated allogeneic splenocytes (BALB/c stimulators) and naive $C D 4{ }^{+} C D 25-$ responder cells. Proliferation was assessed after 5 days. $n=4-6$ mice per group from 2 independent experiments. No statistical difference between treated and naive mice was observed at any cell ratio.

enhanced Treg/DC interactions in vivo, promoting NFAT activation, which was required for Treg expansion. Collectively, these findings demonstrate novel cellular and molecular mechanisms by which Tregs can be expanded in vivo for therapeutic purposes.

\section{Results}

Anti-CD45RB induces FOXP3+ Tregs in vivo. Since allograft survival mediated by anti-CD45RB is dependent on CD25+ cells and anti-CD45RB augments CTLA-4 expression (27-29), we asked whether anti-CD45RB induces Tregs in vivo. As previously shown, anti-CD45RB increased the percentage of $\mathrm{CD}^{+}$cells expressing CTLA-4 almost 2-fold (Figure 1A). We now show that antiCD45RB also significantly augmented the frequency of splenic CD4 cells expressing FOXP3 from an average of $13.8 \%$ to $21.3 \%$ on day 10 (Figure 1A). This occurred without depletion of CD4 ${ }^{+}$ Tconvs (Figure $1 \mathrm{C}$ and Figure 2B). Notably, anti-CD45RB increased FOXP3 expression in both alloantigen-exposed mice and naive mice, indicating that the expansion occurred independently of exogenous antigen (Figure 1A). The increased CD25 and CTLA-4 expression on $\mathrm{CD}^{+}$cells in response to anti-CD45RB occurred entirely on $\mathrm{FOXP3}^{+}$cells, which, for the most part (70\%-80\%), coexpressed these molecules (Figure 1A). Treatment with control IgG $\mathrm{mAb}$ had no effect on Tregs or FOXP3.

The increased percentage of $\mathrm{FOXP}{ }^{+} \mathrm{CD} 4^{+}$cells induced by anti-CD45RB was associated with a similar1.6-to1.9-fold increase in the absolute number of $\mathrm{FOXP}^{+}, \mathrm{CD} 25^{+}$, and CTLA $-4^{+} \mathrm{CD} 4^{+}$ cells in spleens (Figure 1B) and lymph nodes (data not shown).
Treg numbers returned to baseline by day 14 (data not shown). This increase in Tregs occurred without affecting surface expression of activation markers on $\mathrm{FOXP}^{+}$or on $\mathrm{FOXP}^{-}$cells (Figure 1D), whereas anti-CD45RB decreased CD45RB expression on $\mathrm{CD}^{+}$(Tconv) cells, as previously described (25). Moreover, $\mathrm{CD} 4{ }^{+} \mathrm{CD} 25^{+}$cells sorted from untreated and anti-CD45RBtreated mice were equally potent on a per cell basis at suppressing $\mathrm{CD}^{-} 5^{-} \mathrm{T}$ cells in vitro (Figure 1E). These data suggest that the increased $\mathrm{FOXP} 3{ }^{+} \mathrm{CD} 4{ }^{+}$cells, induced by anti-CD $45 \mathrm{RB}$, are fully functional Tregs.

Anti-CD45RB-mediated increase in Tregs occurs in the periphery. Anti-CD45RB could increase Tregs through biologically distinct mechanisms. To rule out an effect on thymic output, we showed that anti-CD45RB increased Treg numbers in euthymic and thymectomized mice equally (Figure 2A). Concordantly, thymectomy did not prevent anti-CD45RB-induced prolongation of islet allograft survival by anti-CD45RB (29). This suggests that antiCD45RB acts on Tregs in the periphery. To confirm this, congenic $\mathrm{CD} 4^{+}$cells were adoptively transferred into replete naive congenic WT mice with or without anti-CD45RB treatment. Consistent with the increase in endogenous $\mathrm{FOXP}^{+}$cells (Figure 1, A and B), anti-CD45RB increased both the percentage (data not shown) and absolute number of transferred $\mathrm{CD} 4^{+}$cells expressing $\mathrm{FOXP}^{+}$ (2.6-fold), without affecting FOXP3- cells (Figure 2B).

CD45 ligation specifically expands $n$ Tregs by enhancing their HP. The increase in Tregs in the periphery induced by anti-CD45RB could have resulted from the de novo induction of iTregs and/or 
A

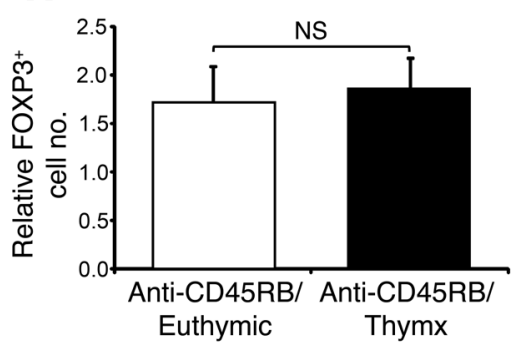

B

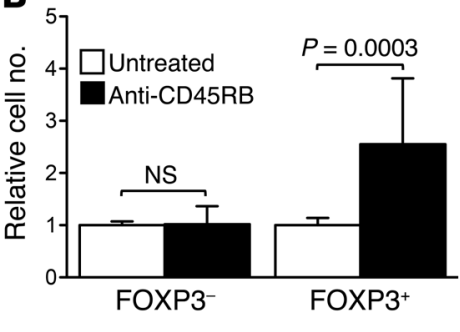

Figure 2. Anti-CD45RB Treg induction occurs independently of thymic output. (A) Number of FOXP3 ${ }^{+}$CD4 ${ }^{+}$cells induced by anti-CD45RB (spleen, day 10 ) in euthymic or thymectomized animals relative to that of naive untreated mice ( $n=5$ mice per group in 2 independent experiments). (B) Number of FOXP3-CD4 ${ }^{+}$or FOXP3 ${ }^{+} \mathrm{CD} 4^{+}$cells recovered in spleens of congenic recipients 10 days after adoptive transfer of $C D 4^{+} T$ cells into untreated and anti-CD45RB-treated mice ( $n=10$ mice per group from 4 independent experiments). undergoing rapid $\mathrm{HP}$, with a near doubling of the fraction that underwent $>7$ divisions after antiCD45RB treatment (31\% $\pm 7 \%)$, compared with that after control IgG $(18 \% \pm 6 \%$; Figure $4 \mathrm{~A})$. Accordingly, the proliferation and replication indexes of transferred nTregs were significantly increased upon anti-CD45RB treatment, compared with those upon treatment with control IgG (Supplemental Figure 1A; supplemental material available online with this article; doi:10.1172/JCI74087DS1). Overall, this led to a 2.3-fold increase in number of transferred $\mathrm{FOXP3}^{+}$cells and a 3-fold increase in number of Tregs undergoing proliferation (Figure 4B). AntiCD45RB also significantly increased the number of nonproliferating Tregs recovered (Figure 4B). However, on average, $74 \%$ of the increase in number of Tregs after CD45 ligation occurred in proliferating the expansion of nTregs. To determine whether anti-CD45RB induces conversion of Tconvs into iTregs, FOXP3- Tconvs were sorted from FOXP3-red fluorescent protein reporter knockin mice (Foxp3-RFP mice) (Figure 3A), stained with CFSE, and transferred into naive WT congenic animals that were then treated with control IgG or anti-CD45RB. Anti-CD45RB treatment had no effect on the frequency (Figure 3B) or on the number of cells newly expressing $\mathrm{FOXP3}^{+}$(Figure 3C), indicating that antiCD45RB does not induce conversion of Tconvs into iTregs. Of note, anti-CD45RB had no effect on the proliferation of Tconvs (data not shown and Figure 4, D and E).

To assess how anti-CD45RB affects nTregs, CFSE-stained, sort-purified $\mathrm{FOXP}^{+}$cells from Foxp3-RFP mice (Figure 3A) were transferred into naive WT congenic animals, using a procedure analogous to that described above. In steady state (IgGtreated controls), $44 \%$ of Tregs underwent HP over 10 days (Figure 4A). Anti-CD45RB significantly enhanced HP of transferred nTregs, with 55\% undergoing proliferation. Of note, antiCD45RB preferentially enhanced the proliferation of the nTregs cells (Figure $4 \mathrm{C}$ ). Taken together, our data demonstrate that anti-CD45RB specifically expands nTregs.

These results were corroborated by adoptive transfer of whole $\mathrm{CD}^{+}{ }^{+} \mathrm{T}$ cells from congenic mice into replete $\mathrm{WT}$ animals. Ten days after transfer, anti-CD45RB significantly increased proliferation of Tregs from $39 \%$ (untreated) to $67 \%$ (after anti-CD45RB treatment), resulting in a 4 -fold increase in the number of proliferating Tregs (Figure 4, D and E). Transferred FOXP3- cells (Tconvs) in these animals remained unaffected. Concordantly, anti-CD45RB increased HP of endogenous Tregs, with an average of $32 \%$ incorporating BrdU over 3 days in treated animals compared with $20 \%$ in untreated animals, while HP of endogenous FOXP3- ${ }^{-} \mathrm{CD} 4^{+}$was unaffected (Figure 4F). Thus, Treg HP can be specifically targeted by anti-CD45RB, leading to a significant increase in Treg number.

Based on the increase in nonproliferating cells (Figure 4B), anti-CD45RB may also enhance Treg survival. In this regard, we found that CD45 ligation significantly decreased apoptosis in Tregs harvested from spleens of mice on day 10 (Supplemental Figure 1B). Since TGF- $\beta$ receptor signaling has been shown to
A

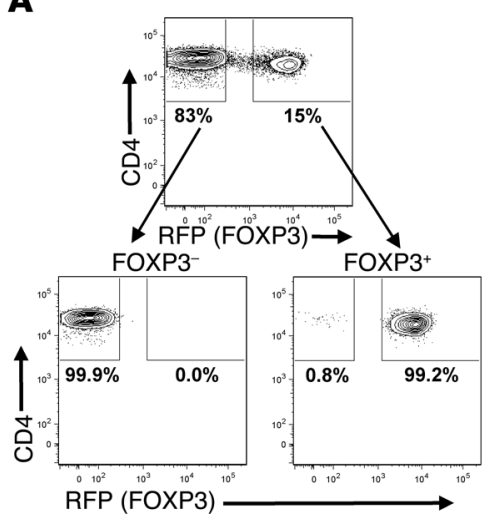

B

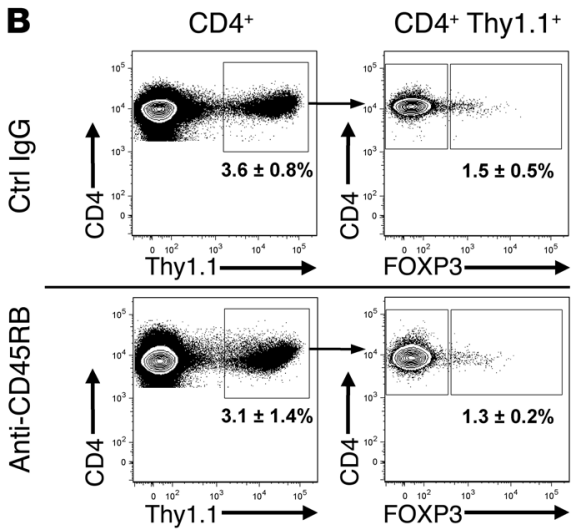

C

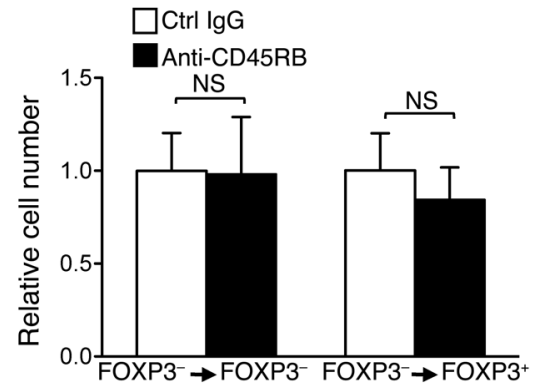

Figure 3. Anti-CD45RB does not induce iTregs in adoptively transferred Tconvs. (A) Representative flow cytometry plots showing sort gates used to isolate FOXP3+ or FOXP3- CD4 ${ }^{+}$cells from Foxp3-RFP congenic (Thy1.1) mice (top row) and purity assessment after sort (bottom row). Numbers represent percentage of events in each gate. (B) Representative flow cytometry plots of live CD4+ cells recovered after adoptive transfer of highly purified FOXP3-CD4+Thy1.1+ cells (obtained as in A) into WT congenic mice that were then treated with control IgC or anti-CD45RB (examined in spleens on day 10). Numbers indicate average $\left( \pm \mathrm{SD}\right.$ ) percentage of transferred (Thy1.1 ${ }^{+} \mathrm{CD} 4^{+}$) cells recovered among total live $\mathrm{CD} 4^{+} \mathrm{B} 22 \mathrm{O}^{-}$cells and $\mathrm{FOXP3}$ expression within the Thy1.1 $1^{+}$-transferred cell population. (C) Number of FOXP3-CD4+ and FOXP3 ${ }^{+}$CD4 ${ }^{+}$cells from from right column in B after anti-CD45RB, relative to control IgC ( $n=9$ mice per group from 3 independent experiments). 
A
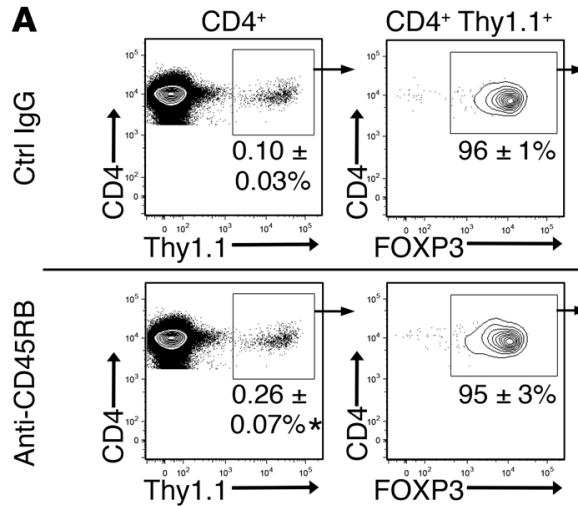

D

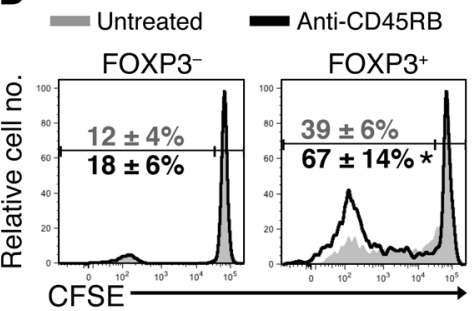

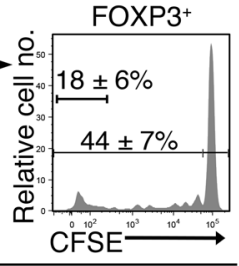

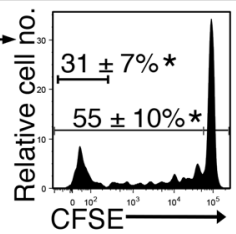

E

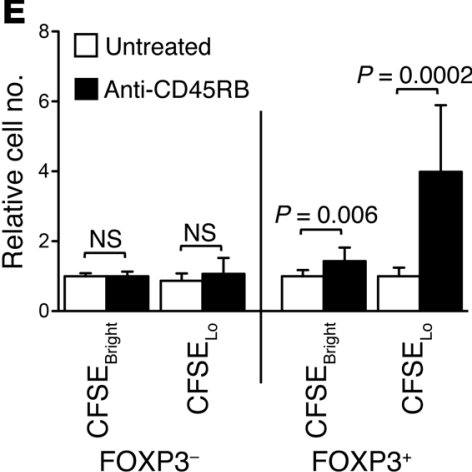

B

C
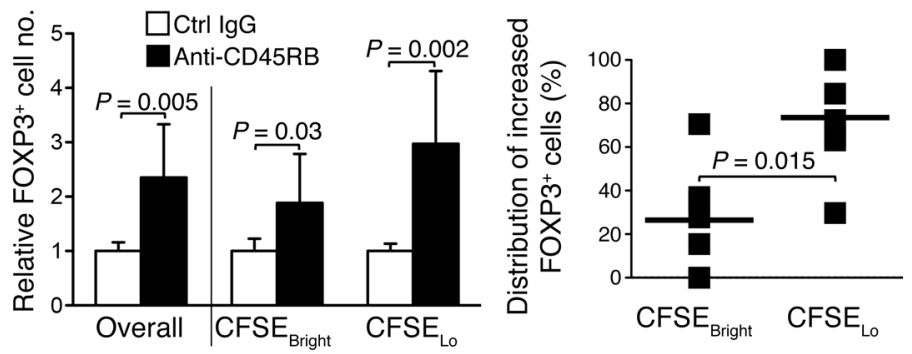

Figure 4. Anti-CD45RB increases Tregs by augmenting HP of $\mathbf{n T r e g s . ~ ( A ) ~ F l o w ~ c y t o m e t r y ~ p l o t s ~ o f ~ l i v e ~ C D 4 + ~ c e l l s ~ r e c o v e r e d ~ a f t e r ~ a d o p t i v e ~ t r a n s f e r ~ o f ~ h i g h l y ~}$ purified FOXP3 ${ }^{+}$CD4 ${ }^{+}$Thy $11^{+}$cells (obtained as in Figure $3 \mathrm{~A}$ ) into control IgG- or anti-CD45RB-treated WT congenic mice (examined in spleen on day 10). Numbers indicate average $\left( \pm \mathrm{SD}\right.$ ) percentage of transferred cells $\left(\right.$ Thy1.1 $\left.+\mathrm{CD} 4^{+}\right)$, FOXP3 expression within transferred cells, and CFSE dilution among FOXP3 ${ }^{+}$-transferred cells ( $n=8$ mice per group, 4 independent experiments). ${ }^{*} P<0.05$ vs. control IgG. (B) Number of recovered FOXP3 ${ }^{+}$cells from right column in A, relative to control lgG. (C) Distribution (CFSE ${ }_{\text {bright }}$ Vs. CFSE $_{10}$ ) of increased FOXP3 ${ }^{+}$cells in response to anti-CD45RB treatment over control IgG from right column in A. Squares represent individual mice, and bars show averages. (D) Flow cytometry plot of CFSE dilution profiles of FOXP3-CD4 ${ }^{+}$ and $\mathrm{FOXP3}^{+} \mathrm{CD}_{4}{ }^{+}$cells recovered after transfer of total $\mathrm{CD} 4^{+} \mathrm{T}$ cells into congenic mice that were untreated or treated with anti-CD45RB (examined in spleens on day 10; $n=10$ mice per group, 4 independent experiments). Numbers represent average $( \pm \mathrm{SD})$ percentage of $C F S E_{10} \cdot{ }^{*} P<0.05$ vs. untreated. (E) Number of $\mathrm{CFSE}_{\text {bright }}$ and $\mathrm{CFSE}_{10}$ cells from D, relative to control IgG. (F) Flow cytometry histograms and average $( \pm \mathrm{SD})$ percentage of BrdU incorporation in endogenous FOXP3 ${ }^{-}$and $\mathrm{FOXP3}^{+} \mathrm{CD}^{+}$cells from untreated and anti-CD45RB-treated mice (day 10). Mice were fed BrdU on days $7-10$ ( $n=4$ mice per group, 2 experiments; ${ }^{*} P=0.01$ vs. untreated).

promote Treg survival in the periphery $(30,31)$, we determined whether this pathway was involved in the anti-CD45RB-mediated increase in Tregs.

We generated mice in which TGF $\beta$ RII could be conditionally deleted by crossing Tgbr $2^{f / f l}$ mice (32) with Ubc-ERT2-Cre mice. Sort-purified CD $25^{+} \mathrm{CD} 4^{+}$cells from $\mathrm{Tg} b r 2^{f / f} \times U b c$-ERT2-Cre mice were adoptively transferred into replete congenic mice. Recipients were then treated with tamoxifen for 5 days to delete TGFßRII. Two days later, mice were treated with either control IgG or anti-CD45RB and assessed on day 10. In both anti-CD45RB and control IgG-treated mice, approximately $85 \%$ of the transferred CD $4^{+}$cells lost TGF $\beta$ RII cell surface expression (data not shown). Transferred cells that remained TGF $\beta \mathrm{RII}^{+}$served as an internal control. Compared with control IgG treatment, anti-CD45RB significantly increased the number of Tregs recovered, regardless of whether they had lost or maintained TGFBRII expression (Supplemental Figure 1C). These data indicated that TGF- $\beta$ signaling is not essential for anti-CD45RB-mediated increase in Tregs.

CD45 ligation does not promote $I L-2 R$ signaling in Tregs. Since Treg homeostasis relies on IL-2R signaling, and CD45 can act as a JAK PTPase (33), we asked whether anti-CD45RB was promoting
Treg proliferation through augmented IL-2R signaling. CD4 cells isolated from mice 10 days after treatment with either control IgG or anti-CD 45RB were exposed to IL-2 ex vivo and stained for pSTAT-5 (34). Reflecting low expression of the high-affinity IL-2R (CD25) in the steady state, a very small fraction ( $2 \%)$ of Tconvs isolated from either IgG control- or anti-CD45RB-treated mice had detectable pSTAT-5 upon IL-2 exposure (Supplemental Figure 2). In contrast, approximately $80 \%$ of Tregs from IgG-treated mice rapidly phosphorylated STAT-5 in the presence of IL-2 (Supplemental Figure 2). However, there was no difference in STAT- 5 phosphorylation in response to IL-2 by Tregs isolated from anti-CD45RB-treated mice. This suggests that anti-CD45RB-induced Treg proliferation in vivo is not due to enhanced IL-2R signaling in Tregs.

CD45 ligation specifically enhances Treg proliferation in response to cognate antigen. The requirement for class II expression on DCs for Treg HP indicates that Treg HP is driven by endogenous antigen (17). To determine whether the presence of cognate antigen is required for anti-CD45RB-mediated proliferation of Tregs, we used TCR transgenic CD $4^{+}$OT-II cells. CFSE-labeled OT-II CD4 ${ }^{+}$ cells $\left(\sim 3 \% \mathrm{FOXP}^{+}\right)$were adoptively transferred into congenic WT recipients that were untreated, treated with anti-CD45RB, immu- 


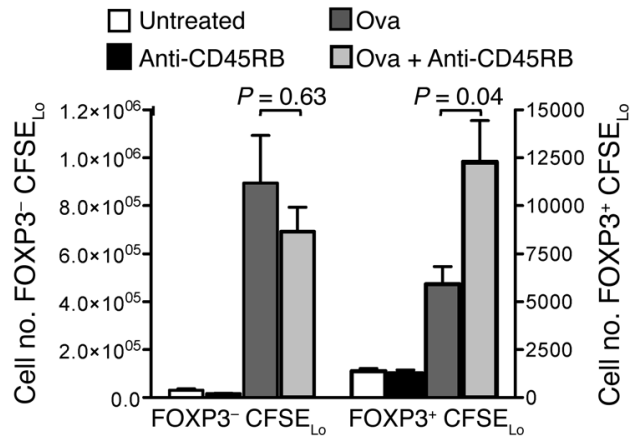

nized with ovalbumin, or treated and immunized. Ovalbumin exposure induced a multifold increase in proliferating OT-II Tregs compared with that in unimmunized mice (day 7; Figure 5). The combination of anti-CD45RB with ovalbumin exposure resulted in a further 2-fold increase in the number of proliferating Tregs, compared with that after exposure to ovalbumin alone (Figure 5). While ovalbumin exposure markedly enhanced proliferation of Tconvs, anti-CD45RB had no further effect. In the absence of cognate antigen, anti-CD45RB had no effect on either Tconv or Treg OT-II cells. Thus, anti-CD45RB specifically expands Tregs in an antigen-dependent and antigen-specific manner.

CD45 ligation preferentially reduces Treg motility, leading to increased Treg contact time with DCs. TCR signaling requires interaction with MHC II antigen complexes, a process that depends on stable T cell/DC interactions resulting from activation of lympho-
Figure 5. Anti-CD45RB augments nTregs response to cognate antigen specifically. Number of FOXP3- ${ }^{-} D 4^{+} \mathrm{CFSE}_{10}$ and $\mathrm{FOXP3}^{+} \mathrm{CD} 4^{+} \mathrm{CFSE}_{10} \mathrm{OT}-\mathrm{II}$ $T$ cells recovered after adoptive transfer of OT-II CD4 ${ }^{+} T$ cells into WT congenic mice that were untreated or treated with anti-CD45RB, ovalbumin (Ova), or ovalbumin and anti-CD45RB. Average \pm SEM is shown $(n=3-7$ mice per group from 3 independent experiments).
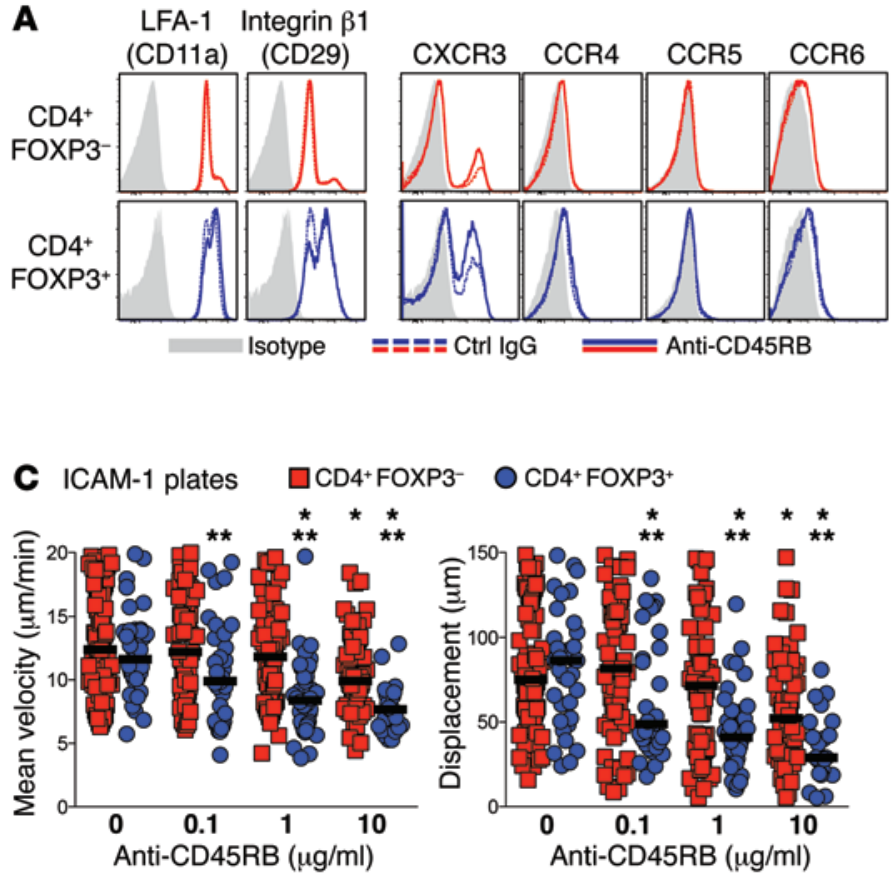

cyte function antigen-1 (LFA-1) on T cells through "inside-out" signaling (35-38). This led us to ask whether anti-CD45RB could affect Treg motility, promote Treg/DC interactions, and preferentially enhance Treg antigen responsiveness in vivo. First, we examined the effect of anti-CD45RB on surface expression of representative chemokine receptors and integrins. While anti-CD45RB had no effect on chemokine receptors CCR4, CCR5, or CCR6, it increased CCCR3 on both Tconvs and Tregs (Figure 6A). LFA-1 (CD11a) and integrin $\beta 1$ (CD29) are well expressed on both Tregs and Tconvs, with a slightly higher level of expression observed on Tregs. AntiCD45B induced a marginal increase in the expression of both CD11a and CD29 on Tregs. However, increased surface expression does not necessarily correlate with the high-affinity conformation necessary for firm binding of integrins to their ligands. Therefore, we addressed this using in vitro motility assays described below.

\section{B ICAM-1 plates}

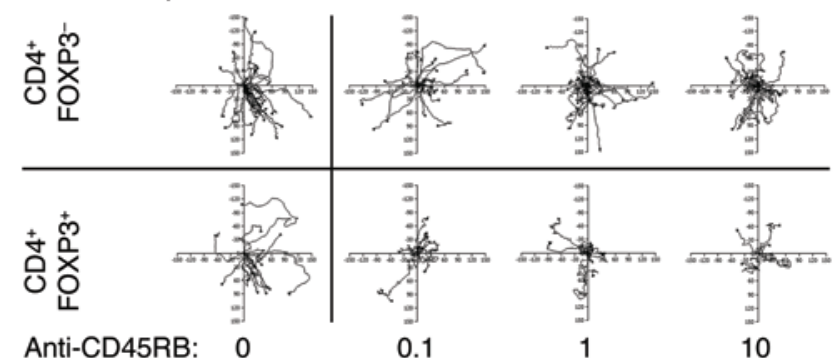

$(\mu \mathrm{g} / \mathrm{ml})$

\section{Fibronectin plates}

$\square \mathrm{CD}^{+}{ }^{+} \mathrm{FOXP3}^{-} \mathrm{OCD4} 4^{+} \mathrm{FOXP3}^{+}$
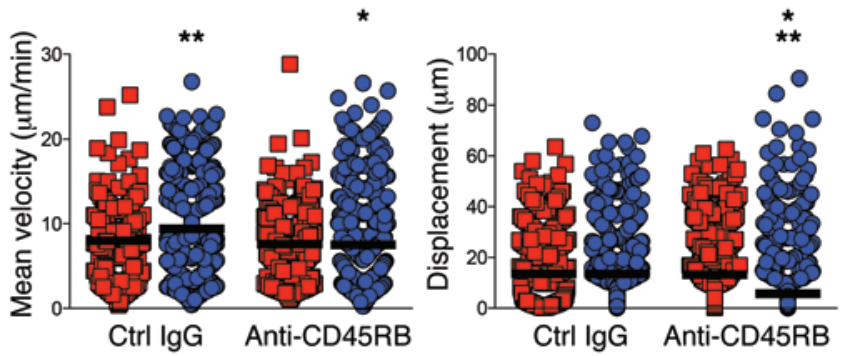

Figure 6. Anti-CD45RB specifically inhibits integrin-dependent motility of Tregs. (A) Representative flow cytometry histograms of surface expression of

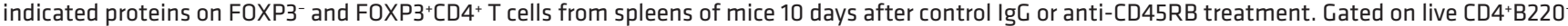
CD8a- lymphocytes. $n=3$ mice per group. (B) Representative cell-tracking diagrams of rested FOXP3- and FOXP3+CD4+ ${ }^{+}$cells on ICAM-1-coated plates with or without the addition of anti-CD45RB in graded concentrations. Axis numbers indicate cell position from origin ( $\mu$ m). (C) Mean velocity and displacement values of the tracks from B. Between 21 and 84 cells were tracked in each group (from 2 independent experiments). A third independent experiment gave similar results (data not shown). (D) Mean velocity and displacement values of rested FOXP3- and FOXP3 ${ }^{+} \mathrm{CD} 4^{+} \mathrm{T}$ cells on fibronectin-coated plates with the addition of control IgC or anti-CD45RB $(1 \mu \mathrm{g} / \mathrm{ml})$. Between 246 and 1,091 cells were tracked in each group (from 2 independent experiments). Horizontal bars indicate median values. ${ }^{*} P<0.05$ vs. untreated or control IgG, ${ }^{* *} P<0.05$ vs. FOXP3 ${ }^{-C D 4}{ }^{+}$for each condition. 
A

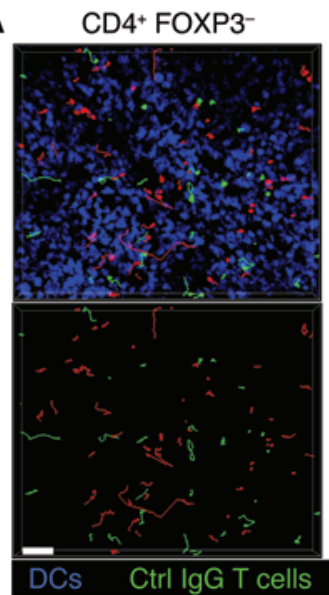

D

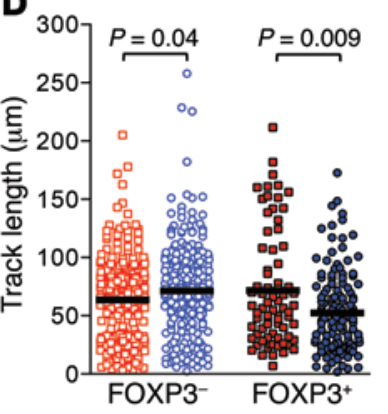

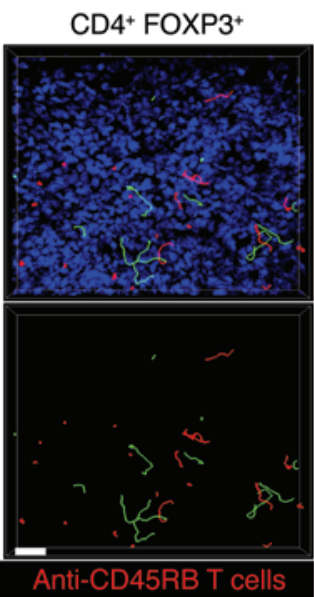

$\mathbf{E}$

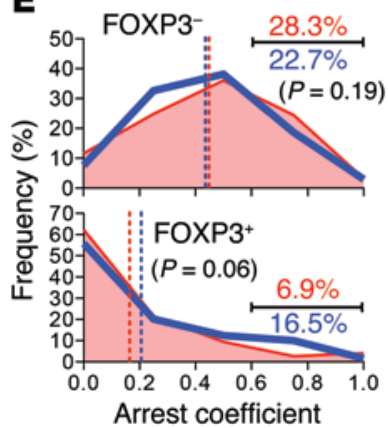

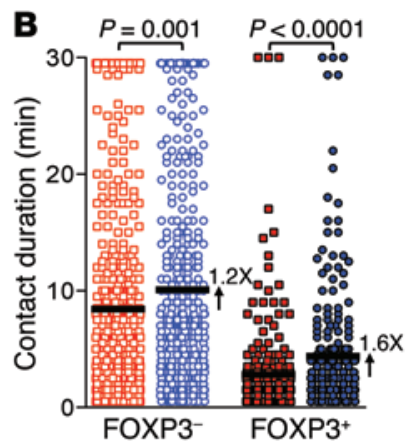

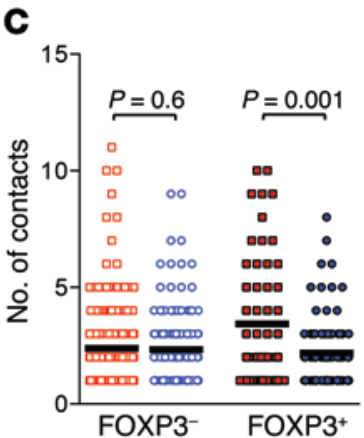

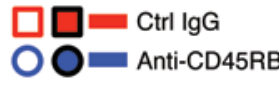

$\mathbf{F}$

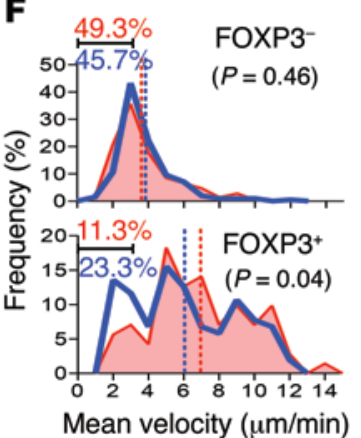

Figure 7. CD45 ligation enhances Treg/DC contacts in vivo. Naive Cd11c-YFP mice received cotransfer of either FOXP3-CD4+ ${ }^{+}$cells isolated on day 4 from mice treated with IgG or anti-CD45RB or FOXP3 ${ }^{+} \mathrm{CD}_{4}{ }^{+}$cells isolated on day 4 from mice treated with IgG or anti-CD45RB. (A) Representative cell tracks from 30-minute time-lapse images acquired by 2PIM of splenic red pulp 1 day after cotransfer of control IgG- or anti-CD45RB-exposed FOXP3-CD4+ or FOXP3 ${ }^{+} \mathrm{CD}^{+}$cells. Images correspond to Supplemental Video 1 . Bottom row shows cell tracks only (scale bar: $50 \mu \mathrm{m}$ ). (B-D) Quantification of motility and T cell/DC interactions from images in A. (B) T cell/DC contact duration time (numbers indicate fold increase vs. control IgC), (C) number of T cell/DC contacts per T cell track, (D) total traveled distance per track. Bars represent mean. (E and F) Cell tracks from images in $\mathbf{A}$ were analyzed (E) for arrest coefficient (fraction of time a T cell has an instantaneous velocity $\leq 2 \mu \mathrm{m} / \mathrm{min}$ over the entire tracking time) and (F) for mean velocity, and plotted as frequency distribution. Vertical dashed lines show mean, and numbers represent percentages of tracks with a value of $\geq 0.6(\mathbf{E})$ and a value of $\leq 3 \mu \mathrm{m} / \mathrm{min}(\mathbf{F})$ within each group. $P$ values compare fractions of control vs. anti-CD45RB-treated CD4 tracks within cutoffs defined above. $n=4$ mice per group from 4 independent experiments. 213 control IgG FOXP3-, 199 anti-CD45RB FOXP3-, 72 control IgG FOXP3', and 103 anti-CD45RB FOXP3+ tracks were analyzed from 11 timelapse images per group.

To examine the effect of anti-CD45RB on LFA-1 adhesion, we assessed the motility of $\mathrm{CD} 4^{+}$cells from Foxp3-GFP (GFP reporter) mice on plates coated with ICAM-1, as we previously reported (39, 40). LFA-1 has a predominant effect on T/DC interactions compared with $\beta 1$ integrins (41). Tregs and Tconvs bound equally to the plates (i.e., $>90 \%$ of cells), while cellular adhesion was almost completely absent in the presence of anti-ICAM- 1 or on uncoated plates (data not shown).

We found that CD 45 ligation preferentially affected motility of Tregs (Figure 6, B and C). While the addition of anti-CD45RB at 1 to $10 \mu \mathrm{g} / \mathrm{ml}$ slowed Tconvs, only $0.1 \mu \mathrm{g} / \mathrm{ml}$ anti-CD $45 \mathrm{RB}$ was necessary to reduce the motility of Tregs, as seen by the restricted migration pattern (Figure 6B). This difference was underscored by measurement of mean velocity and displacement in which a concentration of $10 \mu \mathrm{g} / \mathrm{ml}$ was needed to slow Tconvs to the same degree as that seen for $0.1 \mu \mathrm{g} / \mathrm{ml}$ with Tregs (i.e., 100-fold difference in sensitivity; Figure 6C). Even at the highest dose tested $(10 \mu \mathrm{g} / \mathrm{ml})$, anti-CD $45 \mathrm{RB}$ had a significantly greater effect on motility of Tregs than on that of Tconvs. Anti-CD45RB also exerted a selective slowing of Tregs on fibronectin-coated plates, albeit smaller in magnitude than with LFA-1 (comparing Figure 6C and Figure 6D). This shows that anti-CD45RB also increased adhesion of $\beta 1$ integrins (primarily VLA- 4 and VLA-5). We therefore found that anti-CD45RB preferentially slows integrin-mediated motility of Tregs, and further, this can occur in the absence of concurrent TCR signaling.

Next, we tested the effect of anti-CD45RB on Treg and Tconv motility in vivo using 2-photon intravital microscopy (2PIM). The spleen red pulp was examined because it is both accessible by 2PIM and has an extensive network of DCs, which facilitates the intravital imaging of $\mathrm{DC} / \mathrm{T}$ cell interactions in the steady state (42). FOXP3 ${ }^{+}$Tregs from control IgG or anti-CD45RB-treated Foxp3-RFP mice were sorted on day 4, labeled with distinct fluorescent dyes, and cotransferred into naive syngeneic recipients expressing yellow fluorescent protein (YFP) under the CD11c promoter. Using the same approach, additional sets of mice received cotransfer of FOXP3- Tconvs from control IgG- and from antiCD45RB-treated Foxp3-RFP mice. Twenty-four hours later, the motility of control IgG- and anti-CD45RB-treated Tregs and Tconvs was compared in the spleen using 2PIM over 30-minute- 


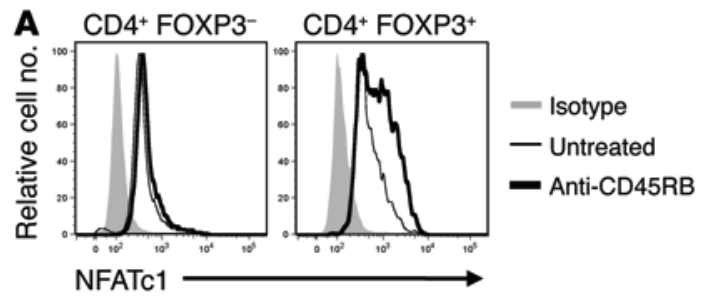

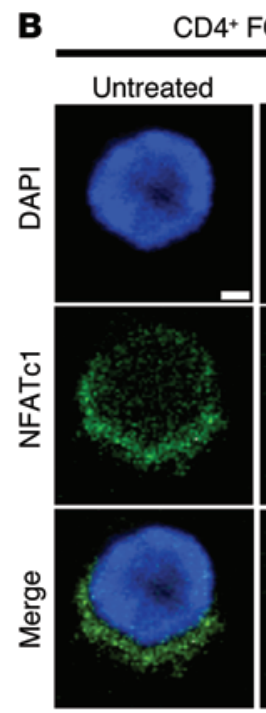

OXP3-

(1)

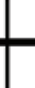

$\mathrm{CD}^{+} \mathrm{FOXP3}^{+}$
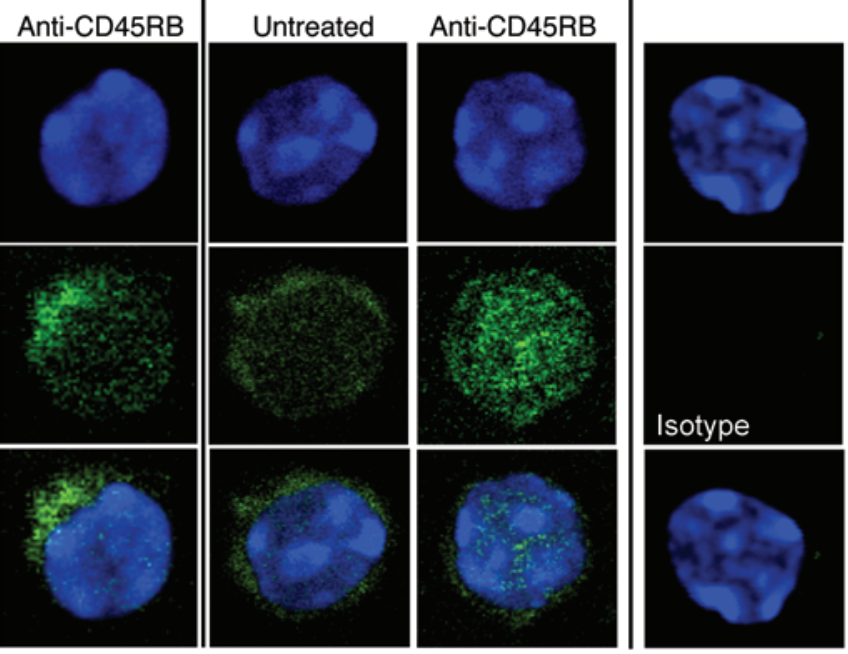

C

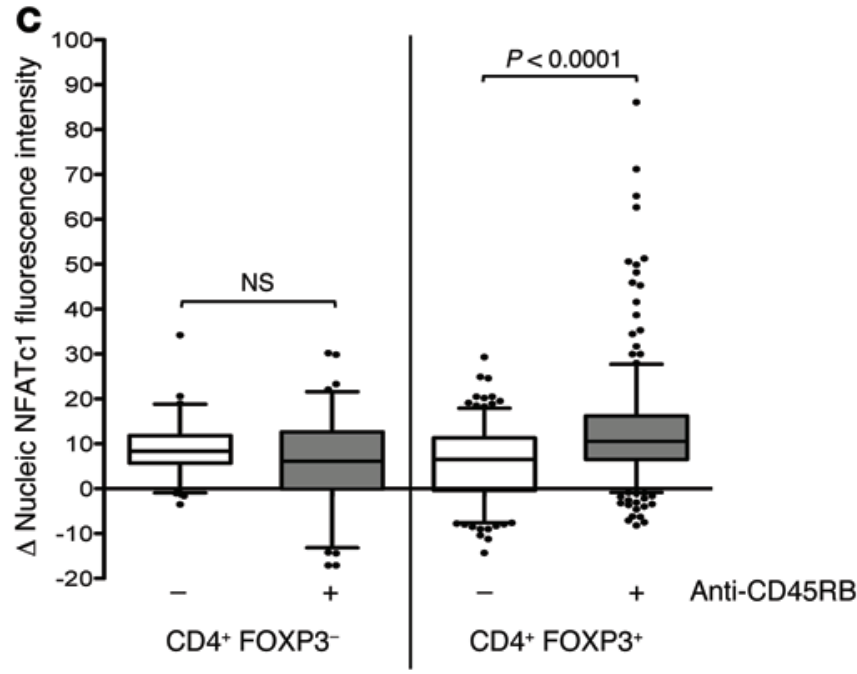

Figure 8. Increased NFAT activation in Tregs after CD45 ligation. (A) Representative NFATc1 immunofluorescence histograms (by flow cytometry) in FOXP3 $-\mathrm{CD}^{+}$and $\mathrm{FOXP3}{ }^{+} \mathrm{CD} 4^{+}$cells from spleens of untreated or anti-CD45RB-treated B6 WT mice (day 7). (B) Representative confocal microscopy images of sorted $\mathrm{FOXP3}^{-}$or $\mathrm{FOXP3}^{+} \mathrm{CD}^{+}$cells from spleens of untreated or anti-CD45RB-treated B6 WT mice on day 7. DAPI was used to stain DNA (scale bar: $2 \mu \mathrm{m}$ ). (C) Box-and-whiskers plot of mean absolute nucleic NFATc1 fluorescence intensity staining from confocal images described in B. Between 47 and 280 individual cells (compiled from 5 to 6 mice in 2 independent experiments) were analyzed in each group. Boxes delineate quartiles, whiskers show 5-95 percentile, and values beyond that range are shown as dots. A third independent experiment gave similar results (data not shown).

long time-lapse images. Of note, this approach examines the behavior of Tregs and Tconvs expressing polyclonal TCRs in absence of exogenous antigen. In steady state (comparing cells from control IgG-treated mice), Tregs traveled faster and spent less time arrested compared with Tconvs, making short (aver- aging 2.8 minutes for Tregs and 8.4 minutes for Tconvs) and more frequent contacts with DCs (Figure 7 and Supplemental Video 1). In comparison to control Tregs, those treated with anti-CD 45 RB exhibited a $60 \%$ increase in contact duration, with fewer DC partners (number of contacts) and a reduced travel distance (Figure 7, A-D, and Supplemental Video 1). Consequently, anti-CD45RB-treated Tregs exhibited a 2 -fold increase in the proportion of cells with an arrest coefficient $\geq 0.6$ or a mean velocity of $\leq 3 \mu \mathrm{m} / \mathrm{min}$ when compared with control IgG-treated Tregs (Figure 7, E and F). In contrast, Tconv behaviors remained mostly unaffected by anti-CD45RB (Figure 7 and Supplemental Video 1). Although an augmentation in the contact duration was detected, the average increase induced by anti-CD $45 \mathrm{RB}$ was only $20 \%$ for Tconvs, compared with $60 \%$ for Tregs (Figure 7B), and there was no change in any other motility parameters. Overall, these data show that CD45 ligation preferentially slowed the motility of Tregs while concurrently increasing Treg/DC contact times.

CD45 ligation increases NFAT activation in Tregs. The increase in contact times would be expected to generate an increase in signaling that leads to the observed expansion of Tregs. To assess this, the effect of anti-CD45RB on NFAT translocation into the nucleus was examined (Figure 8). NFATc1 expression and localization were evaluated in Tconvs and Tregs from untreated and anti-CD45RBtreated mice using confocal microscopy and flow cytometry. In Tconvs, anti-CD45RB had no effect on either total (flow cytometry; Figure $8 \mathrm{~A}$ ) or nuclear (confocal imaging; Figure 8, B and C) NFATc1 expression as compared with that in untreated mice. In contrast, anti-CD45RB significantly increased both total and nuclear NFATc1 expression in Tregs (Figure 8). Consistent with increased Treg/ DC dwell times, this observation suggests that anti-CD45RB augments Treg proliferation by enhancing TCR-driven NFAT nuclear translocation. As a functional correlate, the calcineurin inhibitor cyclosporin A (CsA) significantly reduced both the absolute number and $\mathrm{HP}$ of transferred $\mathrm{FOXP}^{+}$Tregs compared with that in untreated animals, and these were not restored to baseline by the addition of anti-CD45RB (Figure 9). This effect could be attributable to CsA-mediated inhibition of IL-2 secretion by Tconv, which is essential for Treg homeostasis (14, 21). To address this, mice treated with CsA and anti-CD $45 R B$ also received daily recombinant IL-2 (rIL-2) at a dose that augments Treg number and HP in the absence of CsA (data not 
A

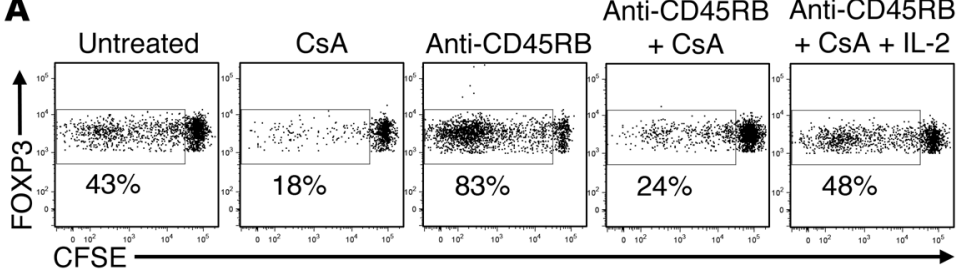

B

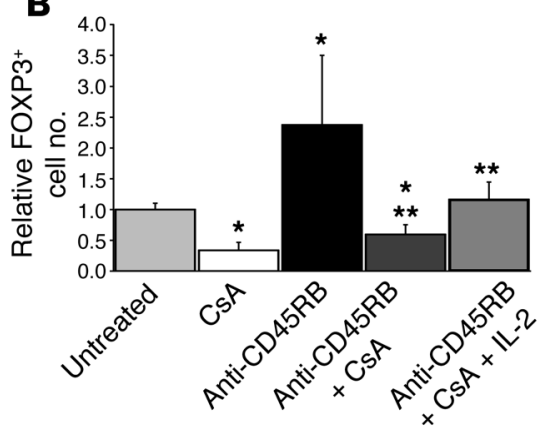

C $\square$ Untreated
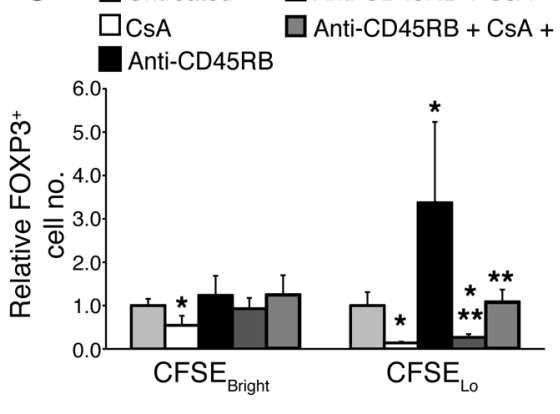

Figure 9. Increased Treg HP by anti-CD45RB depends on intact calcineurin activity. (A) Representative flow cytometry CFSE dilution profiles of $\mathrm{FOXP3}{ }^{+} \mathrm{CD} 4^{+}$cells 10 days after adoptive transfer of congenic CFSE-stained $\mathrm{CD} 4^{+} \mathrm{T}$ cells in spleens of mice that were untreated or treated with CsA, anti-CD45RB, anti-CD45RB and $\mathrm{Cs} A$, or anti-CD45RB, CsA, and rIL-2.

Numbers show representative percentage of events in each gate. (B) Number of transferred $\mathrm{FOXP3}^{+}{ }^{+} \mathrm{CD} 4{ }^{+}$cells (relative to untreated mice) in spleens 10 days after treatment as in $\mathbf{A}$ ( $n=5-8$ mice per group). (C) Number of transferred $\mathrm{FOXP3}^{+} \mathrm{CD} 4^{+}$cells that have proliferated ( $\mathrm{CFSE}_{10}$ ) or have not proliferated ( $\mathrm{CFSE}_{\text {bright }}$ ) in spleens 10 days after treatment as in $\mathbf{A}$ (relative to untreated mice). ${ }^{*} P<0.05$ vs. untreated, ${ }^{* *} P<0.05$ vs. anti-CD45RB. shown). While rIL-2 restored Treg number and HP to baseline levels observed in untreated mice, they remained below that observed in mice treated with anti-CD45RB alone (Figure 9). This suggests that NFAT activation plays an essential role in anti-CD45RB-mediated Treg expansion. Concordantly, CsA has also been shown to interfere with tolerance induction by antiCD45RB (28). Taken together, our data indicate that CD45 ligation enhances Treg conjugate formation with DCs, promotes TCR signaling, and enhances antigen-driven expansion of Tregs in vivo.

\section{Discussion}

Despite their key role in establishing and maintaining tolerance to self-antigens and foreign antigens, the best approach toward expanding Tregs for clinical application remains uncertain. The transfer of ex vivo-expanded Tregs in solid organ transplantation and autoimmunity faces practical limitations, including a requirement for large numbers of cells, possibly infused repeatedly, to try to effect a significant increase in Tregs $(6,43-46)$. In comparison, a reagent-based therapeutic approach for in vivo expansion of Tregs would be more practical. Although targeting Treg expansion in vivo has been described previously, these approaches appear nonspecific in the face of an ongoing immune response (22-24) or expanding CD25 $5^{\text {lo }}$ Tregs in a non-antigen-specific manner by enhancing their IL-2 sensitivity with anti-TNFRSF25 (47). We now show that anti-CD45RB expands Tregs in a novel manner by promoting Treg/DC interactions and augmenting antigen-mediated expansion of nTregs in vivo.

In the steady state, nTregs undergo a high rate of turnover, making them particularly sensitive to factors that affect their proliferation or survival $(16,48)$. In this regard, anti-CD45RB alters normal homeostatic constraints, resulting in a significant increase in Treg proliferation. The preferential expansion of nTregs may provide an advantage compared with iTregs, based on their more stable FOXP3 expression and suppressive phenotype, coupled with differences in gene expression and a higher affinity TCR repertoire (2, 49-53). Importantly, anti-CD45RB specifically enhances the response of Tregs, but not of Tconvs, to cognate anti- gen. In agreement, transplant studies suggest that anti-CD45RB promotes antigen-specific Tregs $(25,29,54)$. For example, antiCD45RB generates donor-specific tolerance that is abrogated with depletion of Tregs by anti-CD25. Moreover, CsA prevents Treg expansion by anti-CD45RB (Figure 9) and blocks anti-CD45RBmediated tolerance (28).

As we showed previously (16), a fraction of Tregs undergoes multiple rounds of division, while almost half do not divide during the observation period (Figure 4, A and D). nTregs express a largely distinct TCR repertoire with increased self-reactivity compared with that of Tconvs (55). Presumably, Tregs undergoing more rapid HP exhibit TCRs with higher affinity to endogenous antigens or antigens from gut flora. Our findings suggest that CD45 ligation preferentially increases proliferation of Tregs already responding to antigen since the nonproliferating pool is largely unchanged (Figure 4, A and D). This could occur by increasing the efficiency of antigen presentation and may promote outgrowth of Tregs with the highest affinity for foreign antigens and self-antigens. Since thymic-derived nTregs represent the majority (>96\%) of FOXP3 ${ }^{+}$ Tregs present in the periphery, anti-CD45RB is most likely targeting these cells. However, anti-CD45RB may also affect the $4 \%$ of the Treg population induced in the periphery.

Upon initial TCR ligation, LFA-1 undergoes conformational change, resulting in increased affinity for its ICAM-1 ligand (3538). This reduces $\mathrm{T}$ cell motility and promotes stable conjugates with APCs. Longer contact times increase the efficiency of $\mathrm{T}$ cell activation by providing time for recruitment of additional MHCpeptide complexes into the contact region (56). In this regard, FOXP3 was shown to induce expression of the adhesion molecule, Neuropilin-1, which augments Treg contact time with DCs and promotes their activation compared with Tconvs (56). We now show that anti-CD45RB preferentially reduces the motility of Tregs, leading to a 2-fold increase in the number of Tregs that arrested their movement, resulting in more prolonged interactions with fewer DCs (Figure 7, B and C). This, in turn, was correlated with increased downstream signaling, as evidenced by enhanced nuclear translocation of NFAT in Tregs. For exam- 
ple, 32\% of Tregs from anti-CD45RB-treated animals expressed nuclear NFAT equal or above that of the top $5 \%$ of Tregs from untreated mice (Figure 8C). NFAT can promote transcription of genes directly involved in proliferation and transcriptional activity of FOXP3, which may itself be involved in Treg homeostasis and enhanced survival (57). In support, we show that NFAT signaling plays a role in anti-CD45RB-mediated Treg proliferation (Figure 9). These anti-CD45RB-mediated changes act predominantly through an LFA-1-dependent pathway, and this occurred in the absence of concurrent TCR engagement (Figure 6). In this regard, previous work by Sakaguchi and colleagues showed a reduction of in vitro aggregation of Tregs on DCs in absence of LFA-1 binding (58). Together, our data indicate that differential regulation of LFA- 1 adhesion, as well as $\beta 1$ integrins, by Tregs and Tconvs is subject to therapeutic manipulation.

CD45 plays a critical role in TCR signaling by regulating the phosphorylation of LCK and FYN protein tyrosine kinases (PTKs). Depending on the tyrosine residue dephosphorylated, CD45 can either increase or decrease PTK activity, and this may depend on timing and colocalization with different pools of substrate (59-62). As a further complexity, the role of the different CD45 isoforms remains enigmatic. Further study will be required to determine how ligation of CD45RB specifically affects signaling leading to increased LFA-1 adhesion, resulting in altered effective Treg/DCs interactions and enhanced expansion. While this could relate to differences in CD45RB expression between Tregs $\left(C D 45 R^{\mathrm{lo}}\right)$ and naive Tconvs $\left(C D 45 R B^{\text {hi }}\right)$, this would not explain why anti-CD45RB did not enhance proliferation of activated Tconvs $\left(C D 45 R^{\mathrm{lo}}\right)$ (63). In this regard, Tregs and Tconvs exhibit differences in the regulation of their interactions with APCs. For example, we showed recently that, in contrast to Tconv/APC conjugation, Treg/APC conjugation is not inhibited by CTLA-4 signaling $(39,40)$. Our current data are the first to demonstrate that such differences in regulation of APC conjugation between Tregs and Tconvs can be targeted for therapeutic purposes.

Despite intense focus on Treg biology, studies assessing how these cells can be specifically induced through intrinsic biological processes to promote tolerance are limited. Our study unexpectedly uncovered that CD45 ligation can rapidly increase Tregs in vivo by promoting antigen-mediated expansion of nTregs. It is now clear that Treg/APC interactions are differentially regulated from Tconv/APC interactions and may be specifically targeted to expand Treg numbers. Further dissection of the signals linking CD45 to Treg HP, "inside-out" signals, and survival will provide new insight and allow identification of additional therapeutic targets. However, this discovery may allow direct therapeutic expansion of Tregs in vivo and may be useful to augment the relative expansion of cultured Tregs after transfer.

\section{Methods}

Animals. Sex-matched 6- to 10-week-old BALB/c (H-2d), C57BL/6 (B6; H-2b), B6.PL-Thy1a/Cy (Thy1.1), B6.SJL-Ptprc ${ }^{a}$ Pepc ${ }^{b} /$ BoyJ (CD45.1), B6.Cg-Tg(Itgax-Venus)1Mnz/J (Cd11c-YFP), B6.Cg-Tg(UbcERT2-Cre)1Ejb/J (Ubc-ERT2-Cre), B6.Cg-FOXP3tm2Tch/J (Foxp3GFP), and B6.Cg-Tg(TcraTcrb) $425 \mathrm{Cbn} / \mathrm{J}$ (OT-II) mice from the National Cancer Institute or The Jackson Laboratory were used. Foxp3RFP knockin mice (B6, $\mathrm{H}-2 \mathrm{~b})$ were generated as described previously
(64). $\operatorname{Tg} b r 2^{f / f l}$ mice were generated as described previously (30, 32) and were crossed with Ubc-ERT2-Cre mice in our facility.

Antibodies and immunofluorescence. Purified anti-CD45RB mAb (MB23G2) and control rat IgG2a were from BioXCell. Biotin or fluorochrome-conjugated mAbs against CD4, CD25, CD19, CD45RB, CTLA-4, FOXP3, CD44, CD45.1, CD62L, CD69, CD90.1, CD90.2, B220, CD11b, CD16/32, CD8, CD11a, CD29, CCCR3, CCR4, CCR5, CCR6, mouse IgG, BrdU, pSTAT-5, annexin V, and Ig control antibodies were from BD Biosciences and eBioscience; anti-NFATc1 mAb (7A6) was from Santa Cruz Biotechnology and Biolegend, and antiTGFßRII was from R\&D Systems. Dead cells were labeled using Fixable Dead Cell Stain (Invitrogen). CD45RB expression was analyzed by staining with directly conjugated anti-CD45RB (clone 16A), which does not cross-react with the epitope bound by MB23G2 $(25,28)$. Negative controls used appropriate Ig fluorochrome conjugates. Flow acquisition was performed on LSRII Fortessa, LSRII, or FACSCalibur analyzers (BD Biosciences), and data were analyzed using Flowjo software (Tree Star). $1 \times 10^{6}$ to $10 \times 10^{6}$ events were acquired per sample. Quantitative cell numbers were calculated according to total live cell counts recovered from individual lymphoid compartments.

Invitro suppression assays. 10 days after initial treatment, $\mathrm{CD} 25^{+} \mathrm{CD} 4^{+}$ cells were isolated from spleens and lymph nodes of $\mathrm{B} 6$ recipients by cell sorting (FACSAria, BD Biosciences). These $\mathrm{CD} 4^{+} \mathrm{CD} 25^{+}$cells were examined for their ability to inhibit $10^{5}$ fresh $\mathrm{CD} 4^{+} \mathrm{CD} 25^{-} \mathrm{B} 6$ responders stimulated with $2 \times 10^{5}$ irradiated BALB/c splenocytes. Proliferation was assessed on day 5 by $\mathrm{H}^{3}$-thymidine uptake.

Thymectomy. As indicated, mice underwent thymectomy as described previously (29). Briefly, a plastic tip was introduced into the mediastinum through a sternotomy, and the thymus was removed by suction. Successful thymectomy was reassessed at the time of sacrifice.

Treatment protocols. Mice received 3 doses of anti-CD45RB (MB23G2; $100 \mu \mathrm{g}$; i.v.) on days 0,1 , and $5(25,28)$. Control recipients were untreated or treated with control IgG using the same dosage. Some recipients were exposed to alloantigen by i.p. injections of $25 \times 10^{6}$ splenocytes from BALB/c mice or by transplanting 200 $\mathrm{BALB} / \mathrm{c}$ islets under the left kidney capsule on day 0 , as we previously described (65). Thymectomy was performed on day -7. CsA was given i.p. daily from day 0 at $40 \mathrm{mg} / \mathrm{kg} / \mathrm{d}$. Recombinant mouse IL-2 (eBioscience) was injected i.p. daily at $1.5 \mu$ g. Tamoxifen in corn oil (SigmaAldrich) was given at $50 \mathrm{mg} / \mathrm{d}$ i.p.

Adoptive cell transfer. $\mathrm{CD}^{+}$cells from spleens and lymph nodes of B6 Thy1.1 mice were enriched by negative selection using BioMag beads (Qiagen) or EasySep (Stem Cell Technologies), stained with CFSE (2.0$2.5 \mu \mathrm{M}$ 5-(and-6)-carboxyfluorescein diacetate, succinimidyl ester; Invitrogen) as indicated, and transferred into sex-matched B6 (Thy1.2) mice on day $-1\left(10 \times 10^{6}\right.$ to $30 \times 10^{6}$ cells per recipient $)$. $\mathrm{CD}^{+}{ }^{+} \mathrm{RFP}^{-}$or $\mathrm{CD}^{+} \mathrm{RFP}^{+}$cells from spleens and lymph nodes of Foxp3-RFP mice (B6; Thy1.1) were sorted using a FACSAria cell sorter (BD Biosciences). In a given experiment, equal numbers of $\mathrm{RFP}^{-}$cells (range $9 \times 10^{6}$ to $10 \times 10^{6}$; $99.9 \%$ pure) or equal numbers of $\mathrm{RFP}^{+}$cells (range $2 \times 10^{6}$ to $3 \times 10^{6}$; 99.2\% pure) were injected i.v. into sex-matched B6 Thy1.2 mice on day-1 and left untreated or treated with control IgG or anti-CD45RB. In separate experiments, highly pure $\mathrm{CD} 4{ }^{+} \mathrm{CD} 25^{+}$cells from spleens and lymph nodes of $T g b r 2^{A / / l} \times U b c-E R T 2-C r e$ mice (B6; Thy1.2) were sorted using a FACSAria cell sorter (BD Biosciences) and transferred into sex-matched B6 CD45.1 mice on day -6 and treated with tamoxifen (on days -6 to -2 ) and anti-CD45RB (days 0, 1, and 5) or treated with tamoxifen and 
control IgG. For 2-photon intravital imaging of spleens, CD4 $4^{+} \mathrm{RFP}^{-}$or $\mathrm{CD} 4^{+} \mathrm{RFP}^{+}$cells from spleens and lymph nodes of Foxp3-RFP mice that received anti-CD45RB or control IgG were sorted on day 4, stained with CellTracker Violet (CTV) or CellTracker Orange (CTO; $2.0 \mu \mathrm{M}$; Invitrogen), and transferred into naive sex-matched Cd11c-YFP mice. Dyes were alternated between experiments.

Ex vivo IL-2R signaling assessment. Splenocytes from B6 mice that received control Ig or anti-CD45RB were exposed to $50 \mathrm{U} / \mathrm{ml} \mathrm{rmIL-2}$ (eBioscience) for 15 minutes prior to fixation/permeabilization and pSTAT-5 staining.

In vitro live cell imaging for motility assays. Bead-enriched (Dynal) $\mathrm{CD}^{+} \mathrm{T}$ cells from Foxp3-GFP mice (used on ICAM-1-Fc-coated plates), or sorted $\mathrm{CD}^{+}{ }^{+} \mathrm{FOXP}^{-}$and $\mathrm{CD}^{+}{ }^{+} \mathrm{FOXP}^{+}{ }^{+} \mathrm{T}$ cells from Foxp3RFP mice (used on fibronectin-coated plates), were activated with plate-bound anti-CD3 and anti-CD28 mAbs for 2 days. Cells were rested overnight before being added to $4 \mu \mathrm{g} / \mathrm{ml}$ ICAM-1-Fc- (R\&D Systems) or fibronectin-coated $(20 \mu \mathrm{g} / \mathrm{ml})$ plates and incubated at $37^{\circ} \mathrm{C}$. Cell movement was recorded every 10 seconds for 20 minutes (for ICAM-1-Fc-coated plates) or every 5 seconds for 5 minutes (for fibronectin-coated plates) using Zeiss LSM710 or Nikon Eclipse Ti live cell imaging confocal microscopes, $488 \mathrm{~nm}$ laser excitation, and transmitted light. Data were analyzed using Velocity (Perkin Elmer) or Imaris (Bitplane) softwares. Measurements were performed on adhered cells only, distinguished from nonadhered cells by shape, including cell protrusions.

2PIM. Mice were anesthetized using isoflurane. Spleens of Cd11c-YFP mice were surgically exposed and supported using a custom-made device 1 day after cotransfer of CTO- or CTV-stained CD4 ${ }^{+}$ $\mathrm{RFP}^{-}$(or CD4 ${ }^{+} \mathrm{RFP}^{+}$in separate experiments) T cells from control IgGor anti-CD45RB-treated Foxp3-RFP mice (4 days prior to cell sorting). Splenic blood perfusion, mouse rehydration, and physiological temperature of $37^{\circ} \mathrm{C}$ were preserved and maintained throughout imaging. Two-photon laser scanning imaging was performed using an upright Olympus FV1000 MPE microscope (Olympus) and a Mai Tai DeepSee femtosecond-pulsed Ti:sapphire laser (Spectra-Physics) tuned at $860 \mathrm{~nm}$. The tissue was line scanned, and fluorescence emission was captured by 3 nondescanned external photomultiplier tube detectors coupled to the following band-pass emission filters: $460-500 \mathrm{~nm}$, $520-560 \mathrm{~nm}$, and 570-650 nm. Time-lapse images were acquired using Fluoview software (Olympus). Stacks of 12 optical sections were acquired every 30 seconds for 30 minutes to provide image volumes of $30 \mu \mathrm{m}$ (25- to $55-\mu \mathrm{m}$ deep) in depth and around $450 \mu \mathrm{m}$ in width and height at a resolution of $0.994 \mu \mathrm{m}$ per pixel using a water immer- sion $\times 25$ objective ( $\mathrm{NA}=1.05$; Olympus). Image rendering, tracking of adoptively transferred cells from time sequence of image stacks, motion artifact correction, and masking were performed using Imaris software. Tracks $<2$ minutes were excluded. Each track was inspected manually to ensure accuracy. Motility parameters were generated using Imaris (Bitplane), while contact parameters were measured and compiled manually. Videos were edited using Photoshop CS6 (Adobe).

Nuclear NFATc1 assessment. CD4 ${ }^{+}$T cells from spleens of B6 mice that were left untreated or received anti-CD45RB were enriched and stained for FOXP3 and NFATc1 expression. The DNA of sorted FOXP3 $^{-}$and FOXP3 ${ }^{+}$cells was stained with DAPI (Sigma-Aldrich), and images were acquired by confocal microscopy (Olympus). The nuclei of individual cells were delineated, and the mean fluorescence intensity was measured using ImageJ software (NIH). Mean background fluorescence intensity and mean isotype fluorescence intensity were subtracted from individual values.

Statistics. Nonparametric Mann-Whitney test was used for statistical analyses, except as noted below. The binomial test of proportions was used to address impact of treatment on the fraction of cells with arrest coefficient $\geq 0.6$ (Figure $7 \mathrm{E}$ ) or with mean velocity $\leq 3 \mu \mathrm{m} / \mathrm{min}$ (Figure 7F). Differences were considered to be significant at $P<0.05$. Bar graphs show mean $\pm \mathrm{SD}$, unless otherwise stated in the figure legend.

Study approval. Mice were housed with food and water ad libitum. Studies were performed in compliance with NIH guidelines and approved by the Institutional Animal Care and Use Committee at the University of Pittsburgh.

\section{Acknowledgments}

This work was supported by NIH P01 AI064343 (to D.M. Rothstein, F.G. Lakkis, and W.D. Shlomchik); R01 HL081663 (to P.W. Finn and D.L. Perkins); American Heart Association 0625865T (to G. Camirand); and John Merrill Grant in Transplantation from the American Society of Nephrology (to G. Camirand). We would like to thank the personnel at the Flow Cytometry Facility, the Center for Biologic Imaging, and the Intravital Imaging Facility for their assistance as well as Kun-Wan Chen and Douglas Landsittel for their help and assistance with statistical analysis.

Address correspondence to: David M. Rothstein, Thomas E. Starzl Transplantation Institute, University of Pittsburgh School of Medicine, 1545W BST, 200 Lothrop St., Pittsburgh, Pennsylvania 15261, USA. Phone: 412.648.7154; E-mail: rothsteind@upmc.edu.
1. Sakaguchi S, Yamaguchi T, Nomura T, Ono M. Regulatory T cells and immune tolerance. Cell. 2008;133(5):775-787.

2. Josefowicz SZ, Lu LF, Rudensky AY. Regulatory T cells: mechanisms of differentiation and function. Annu Rev Immunol. 2012;30(1):531-564.

3. Coombes JL, et al. A functionally specialized population of mucosal CD $103^{+}$DCs induces Foxp $3^{+}$regulatory $\mathrm{T}$ cells via a TGF- $\beta$ and retinoic acid-dependent mechanism. J Exp Med. 2007;204(8):1757-1764.

4. Sun C-M, et al. Small intestine lamina propria dendritic cells promote de novo generation of Foxp3 $\mathrm{T}$ reg cells via retinoic acid. J Exp Med.
2007;204(8):1775-1785.

5. Josefowicz SZ, et al. Extrathymically generated regulatory T cells control mucosal $\mathrm{TH} 2$ inflammation. Nature. 2012;482(7385):395-399.

6. Hippen KL, Riley JL, June CH, Blazar BR. Clinical perspectives for regulatory $\mathrm{T}$ cells in transplantation tolerance. Semin Immunol. 2011;23(6):462-468.

7. Gao W, Lu Y, El Essawy B, Oukka M, Kuchroo VK, Strom TB. Contrasting effects of cyclosporine and rapamycin in de novo generation of alloantigen-specific regulatory T cells. Am J Transplant. 2007;7(7):1722-1732.

8. Ochando JC, et al. Alloantigen-presenting plasmacytoid dendritic cells mediate tolerance to vascularized grafts. Nat Immunol. 2006;7(6):652-662.

9. Cobbold SP, et al. Induction of foxP $3^{+}$regulatory $\mathrm{T}$ cells in the periphery of $\mathrm{T}$ cell receptor transgenic mice tolerized to transplants. JImmunol. 2004;172(10):6003-6010.

10. Ferrer IR, Wagener ME, Song M, Kirk AD, Larsen $\mathrm{CP}$, Ford ML. Antigen-specific induced Foxp $3^{+}$ regulatory $\mathrm{T}$ cells are generated following CD40/ CD154 blockade. Proc Natl Acad Sci U S A. 2011;108(51):20701-20706.

11. Kretschmer K, Apostolou I, Hawiger D, Khazaie K, Nussenzweig MC, von Boehmer H. Inducing and 
expanding regulatory $\mathrm{T}$ cell populations by foreign antigen. Nat Immunol. 2005;6(12):1219-1227.

12. Fan Z, et al. In vivo tracking of 'color-coded' effector, natural and induced regulatory $\mathrm{T}$ cells in the allograft response. Nat Med. 2010;16(6):718-722.

13. Hori S, Haury M, Lafaille JJ, Demengeot J, Coutinho A. Peripheral expansion of thymus-derived regulatory cells in anti-myelin basic protein $\mathrm{T}$ cell receptor transgenic mice. Eur J Immunol. 2002;32(12):3729-3735.

14. Setoguchi R, Hori S, Takahashi T, Sakaguchi S. Homeostatic maintenance of natural Foxp3(+) $\mathrm{CD} 25(+) \mathrm{CD} 4(+)$ regulatory $\mathrm{T}$ cells by interleukin (IL)-2 and induction of autoimmune disease by IL-2 neutralization. JExp Med. 2005;201(5):723-735.

15. Lin W, et al. Regulatory $\mathrm{T}$ cell development in the absence of functional Foxp3. Nat Immunol. 2007;8(4):359-368.

16. Tang A, et al. CTLA4 expression is an indicator and regulator of steady-state $\mathrm{CD} 4^{+} \mathrm{FoxP}^{+} \mathrm{T}$ cell homeostasis. JImmunol. 2008;181(3):1806.

17. Darrasse-Jèze G, et al. Feedback control of regulatory $\mathrm{T}$ cell homeostasis by dendritic cells in vivo. J Exp Med. 2009;206(9):1853-1862.

18. Tang $Q$, et al. Cutting edge: $C D 28$ controls peripheral homeostasis of $\mathrm{CD}_{4}{ }^{+} \mathrm{CD} 25^{+}$regulatory T cells. J Immunol. 2003;171(7):3348-3352.

19. Salomon B, et al. B7/CD28 costimulation is essential for the homeostasis of the $\mathrm{CD} 44^{+} \mathrm{CD} 25^{+}$ immunoregulatory $\mathrm{T}$ cells that control autoimmune diabetes. Immunity. 2000;12(4):431-440.

20. Zhang R, Huynh A, Whitcher G, Chang J, Maltzman JS, Turka LA. An obligate cellintrinsic function for CD28 in Tregs. J Clin Invest. 2013;123(2):580-593.

21. Fontenot JD, Rasmussen JP, Gavin MA, Rudensky AY. A function for interleukin 2 in Foxp3expressing regulatory T cells. Nat Immunol. 2005;6(11):1142-1151.

22. Webster KE, et al. In vivo expansion of T reg cells with IL-2-mAb complexes: induction of resistance to EAE and long-term acceptance of islet allografts without immunosuppression. J Exp Med. 2009;206(4):751-760.

23. Chen CY, et al. IL-2 simultaneously expands Foxp $3+\mathrm{T}$ regulatory and $\mathrm{T}$ effector cells and confers resistance to severe tuberculosis (TB): implicative Treg-T effector cooperation in immunity to TB. J Immunol. 2012;188(9):4278-4288.

24. Tomala J, Chmelova H, Mrkvan T, Rihova B, Kovar M. In vivo expansion of activated naive $\mathrm{CD} 8^{+} \mathrm{T}$ cells and NK cells driven by complexes of IL-2 and anti-IL-2 monoclonal antibody as novel approach of cancer immunotherapy. J Immunol. 2009;183(8):4904-4912.

25. Basadonna GP, et al. Antibody-mediated targeting of CD 45 isoforms: a novel immunotherapeutic strategy. Proc Natl Acad Sci U S A. 1998;95(7):3821-3826.

26. Sho M, et al. Requirements for induction and maintenance of peripheral tolerance in stringent allograft models. Proc Natl Acad Sci U S A. 2005;102(37):13230-13235.

27. Ariyan C, et al. Cutting edge: transplantation tolerance through enhanced CTLA-4 expression. JImmunol. 2003;171(11):5673-5677.

28. Fecteau S, Basadonna GP, Freitas A, Ariyan C, Sayegh MH, Rothstein DM. CTLA-4 up-regula- tion plays a role in tolerance mediated by CD 45 . Nat Immunol. 2001;2(1):58-63.

29. Salvalaggio PR, et al. Antigen exposure during enhanced CTLA-4 expression promotes allograft tolerance in vivo.J Immunol. 2006;176(4):2292-2298.

30. Li MO, Wan YY, Flavell RA. T cell-produced transforming growth factor-beta1 controls $\mathrm{T}$ cell tolerance and regulates Th1- and Th17-cell differentiation. Immunity. 2007;26(5):579-591.

31. Wan YY, Flavell RA. 'Yin-Yang' functions of transforming growth factor-beta and $\mathrm{T}$ regulatory cells in immune regulation. Immunol Rev. 2007;220:199-213.

32. Chytil A, Magnuson MA, Wright CV, Moses HL. Conditional inactivation of the TGF- $\beta$ type II receptor using Cre:Lox. Genesis. 2002;32(2):73-75

33. Irie-Sasaki J, et al. CD 45 is a JAK phosphatase and negatively regulates cytokine receptor signalling. Nature. 2001;409(6818):349-354

34. Krutzik PO, Nolan GP. Intracellular phospho-protein staining techniques for flow cytometry: monitoring single cell signaling events. Cytometry A. 2003;55(2):61-70.

35. Dustin ML. Modular design of immunological synapses and kinapses. Cold Spring Harb Perspect Biol. 2009;1(1):a002873.

36. Negulescu PA, Krasieva TB, Khan A, Kerschbaum HH, Cahalan MD. Polarity of T cell shape, motility, and sensitivity to antigen. Immunity. 1996;4(5):421-430.

37. Dustin ML, Bromley SK, Kan Z, Peterson DA, Unanue ER. Antigen receptor engagement delivers a stop signal to migrating T lymphocytes. Proc Natl Acad Sci U S A. 1997;94(8):3909-3913.

38. Mempel TR, Henrickson SE, Von Andrian UH. T-cell priming by dendritic cells in lymph nodes occurs in three distinct phases. Nature. 2004;427(6970):154-159.

39. Lu Y, Schneider H, Rudd CE. Murine regulatory T cells differ from conventional $\mathrm{T}$ cells in resisting the CTLA-4 reversal of TCR stop-signal. Blood. 2012;120(23):4560-4570.

40. Schneider $\mathrm{H}$, et al. Reversal of the TCR stop signa by CTLA-4. Science. 2006;313(5795):1972-1975.

41. Grakoui A, et al. The immunological synapse: a molecular machine controlling $\mathrm{T}$ cell activation. Science. 1999;285(5425):221-227.

42. Waite JC, et al. Dynamic imaging of the effector immune response to listeria infection in vivo. PLoS Pathog. 2011;7(3):e1001326.

43. Tang Q, Bluestone JA, Kang SM. CD4(+)Foxp3(+) regulatory $\mathrm{T}$ cell therapy in transplantation. J Mol Cell Biol. 2012;4(1):11-21.

44. Brennan TV, et al. Requirements for prolongation of allograft survival with regulatory $\mathrm{T}$ cell infusion in lymphosufficient hosts. J Surg Res. 2011;169(1):e69-e75.

45. Tsang JY, et al. Conferring indirect allospecificity on $\mathrm{CD} 4{ }^{+} \mathrm{CD} 25^{+}$Tregs by TCR gene transfer favors transplantation tolerance in mice. J Clin Invest. 2008;118(11):3619-3628.

46. Golshayan D, Jiang S, Tsang J, Garin MI, Motte C, Lechler RI. In vitro-expanded donor alloantigen-specific $\mathrm{CD} 4^{+} \mathrm{CD} 25^{+}$regulatory $\mathrm{T}$ cells promote experimental transplantation tolerance. Blood.2007;109(2):827-835.

47. Schreiber TH, et al. Therapeutic Treg expansion in mice by TNFRSF 25 prevents allergic lung inflammation. JClin Invest. 2010;120(10):3629-3640.

48. Wang Y, et al. Regulatory T cells require mammalian target of rapamycin signaling to maintain both homeostasis and alloantigen-driven proliferation in lymphocyte-replete mice. J Immunol. 2011;186(5):2809-2818.

49. Leguern C. Regulatory $\mathrm{T}$ cells for tolerance therapy: revisiting the concept. Crit Rev Immunol. 2011;31(3):189-207.

50. Huehn J, Polansky JK, Hamann A. Epigenetic control of FOXP3 expression: the key to a stable regulatory T-cell lineage? Nat Rev Immunol. 2009;9(2):83-89.

51. Rubtsov YP, et al. Stability of the regulatory $\mathrm{T}$ cell lineage in vivo. Science. 2010;329(5999):1667-1671.

52. Polansky JK, et al. DNA methylation controls Foxp3 gene expression. Eur J Immunol. 2008;38(6):1654-1663.

53. Hill JA, et al. Foxp3 transcription-factor-dependent and -independent regulation of the regulatory $\mathrm{T}$ cell transcriptional signature. Immunity. 2007;27(5):786-800.

54. Gao Z, et al. Adoptively transferable tolerance induced by CD $45 \mathrm{RB}$ monoclonal antibody. J Am Soc Nephrol. 1999;10(2):374-381.

55. Hsieh CS, Liang Y, Tyznik AJ, Self SG, Liggitt D, Rudensky AY. Recognition of the peripheral self by naturally arising $\mathrm{CD} 25^{+} \mathrm{CD} 4^{+} \mathrm{T}$ cell receptors. Immunity. 2004;21(2):267-277.

56. Sarris M, Andersen KG, Randow F, Mayr L, Betz AG. Neuropilin-1 expression on regulatory T cells enhances their interactions with dendritic cells during antigen recognition. Immunity. 2008;28(3):402-413.

57. Wu Y, et al. FOXP3 controls regulatory $\mathrm{T}$ cell function through cooperation with NFAT. Cell. 2006;126(2):375-387.

58. Onishi Y, Fehervari Z, Yamaguchi T, Sakaguchi S. Foxp $3^{+}$natural regulatory $\mathrm{T}$ cells preferentially form aggregates on dendritic cells in vitro actively inhibit their maturation. Proc Natl Acad Sci U S A. 2008;105(29):10113-10118.

59. Edmonds SD, Ostergaard HL. Dynamic association of CD45 with detergent-insoluble microdomains in T lymphocytes. JImmunol. 2002;169(9):5036-5042.

60. Saunders AE, Johnson P. Modulation of immune cell signalling by the leukocyte common tyrosine phosphatase, CD45. Cell Signal. 2010;22(3):339-348.

61. Wong NK, Lai JC, Birkenhead D, Shaw AS, Johnson P. CD 45 down-regulates Lck-mediated CD44 signaling and modulates actin rearrangement in T cells. J Immunol. 2008;181(10):7033-7043.

62. Hermiston ML, Xu Z, Weiss A. CD45: a critical regulator of signaling thresholds in immune cells. Annu Rev Immunol. 2003;21:107-137.

63. Birkeland ML, Johnson P, Trowbridge IS, Pure E. Changes in CD45 isoform expression accompany antigen-induced murine T-cell activation. Proc Natl Acad Sci U S A. 1989;86(17):6734-6738.

64. Wan YY, Flavell RA. Identifying Foxp3-expressing suppressor T cells with a bicistronic reporter. Proc Natl Acad Sci U S A. 2005;102(14):5126-5131.

65. Salvalaggio PR, et al. Islet filtration: a simple and rapid new purification procedure that avoids ficoll and improves islet mass and function. Transplantation. 2002;74(6):877-879. 